\title{
Caracterização hidráulica de tubos porosos oriundos de pneus reciclados utilizados em irrigação subsuperficial
}

\author{
Marinaldo F. Pinto ${ }^{1}$, Antonio P. de Camargo ${ }^{2}$, Osvaldo Rettore Neto ${ }^{3}$ \& José A. Frizzone ${ }^{4}$ \\ ${ }^{1}$ DE/IT/UFRRJ. Seropédica, RJ. E-mail: mfpufrrj@yahoo.com.br \\ ${ }^{2}$ INCT-EI/ESALQ/USP. Piracicaba, SP. E-mail: apcpires@usp.br (Autor correspondente) \\ ${ }^{3}$ DER/FAEM/UFPel. Pelotas, RS. E-mail: osvaldo.rettore@ufpel.edu.br \\ ${ }^{4}$ INCT-EI/ESALQ/USP. Piracicaba, SP. E-mail: frizzone@usp.br
}

\section{Palavras-chave:}

microirrigação

vazão

perda de carga

\begin{abstract}
R E S U M O
Mesmo diante do apelo ecológico pelo reaproveitamento de resíduos, os produtos de irrigação, como é caso de tubos porosos oriundos de pneus reciclados, devem ser avaliados quanto à sua viabilidade técnica. A caracterização hidráulica é um dos requisitos necessários para tal finalidade além de fornecer dados essenciais ao dimensionamento de sistemas de irrigação. Diante do exposto objetivou-se caracterizar tubos porosos quanto aos fatores perda de carga, vazão em função da pressão e variação de vazão. Foram utilizadas cinco amostras de tubo para determinação da perda de carga e oito para a determinação da curva vazão-pressão e do coeficiente de variação, sendo ensaiados sete níveis de pressão. A perda de carga foi determinada com tubo impermeabilizado e com distribuição de vazão para diferentes vazões. O tubo poroso apresentou coeficiente de variação de vazão em torno de 5\%, considerado resultado satisfatório. O modelo potencial não se ajustou aos dados da curva característica vazão-pressão. Os fatores de atrito e de redução de perda de carga apresentaram comportamento divergente da literatura em que outros fatores, como a variação do diâmetro, devem ser estudados com maiores detalhes.
\end{abstract}

Key words: microirrigation flow rate head loss

\section{Hydraulic characterization of porous pipes made of recycled automobile tires used in subsurface irrigation}

\begin{abstract}
A B S T R A C T
Even considering the ecological appeal to reuse wastes, the technical feasibility of irrigation products, such as porous pipes made of recycled automobile tires, must be assessed. Besides the hydraulic characterization is one of these technical requirements, it also provides essential data for designing subsurface irrigation systems. The objective of the research was to study the following characteristics of porous pipes: head loss, pressure-flow rate relationship, and variation of flow rate. Five samples of pipe were employed for determining head loss; eigth samples were used for determining pressure-flow rate curves and coefficient of variation evaluating seven pressures. Head loss experiments required pipes externally sealed (waterproofed) as well as pipes operating normally under several flow rates. The coefficient of variation of flow was around 5\%, which represents a satisfactory result. The power function did not fit to the experimental data of pressure-flow rate. The head loss coefficient and the friction correction factor due to outflow of emitters presented values which did not match with those found in literature. Effects related to pipe diameter changes due to operating pressure must be taken into account in further studies.
\end{abstract}

\section{INTRODUÇÃo}

Cada vez mais é discutida e incentivada a reutilização de materiais e resíduos visando à sustentabilidade dos recursos naturais. Na agricultura irrigada tem-se percebido algumas iniciativas de reúso de material para concepção de novos produtos, a exemplo dos tubos porosos Osmo-drain fabricados a partir de pneus reciclados. Esses tubos são designados para uso em sistemas de irrigação subsuperficial ou de drenagem (Osmo-Drain, 2012).

O destino de pneus usados é bastante problemático visto serem considerados resíduos sólidos especiais e que, em alguns locais, como na União Europeia, não podem ser enviados para aterros sanitários, mesmo que sejam triturados (Motta, 2008). Um destino possível consiste na reciclagem de pneus cuja borracha pode ser utilizada para fins energéticos, para fabricação de misturas asfálticas e na construção civil, entre outros (Bertollo et al., 2000; Lagarinhos \& Tenório, 2008).

Tubos porosos comercializados para uso em irrigação são produzidos, basicamente, a partir de uma mistura de borracha triturada com partículas de polietileno, que sofrem processo de extrusão dentro de uma câmara cilíndrica sob pressão e temperatura elevadas (Teeluck \& Sutton, 1998). Em geral, a borracha utilizada neste processo provém da reciclagem de pneus usados. 
Apesar do tubo poroso ter começado a ser utilizado recentemente no Brasil, sua utilização em sistemas de irrigação subsuperficial de pomares, jardins e gramados, é relatada há vários anos (Teeluck \& Sutton, 1998). Além de aplicações a campo, Qiaosheng et al. (2007) relatam uma pesquisa que descreve simulações das características do bulbo úmido e o movimento de água no solo quando tubos porosos são utilizados em sistemas de irrigação.

Enquanto linhas laterais convencionais de microirrigação contêm emissores dispostos em espaçamento equidistante, os tubos porosos contêm inúmeras unidades emissoras distribuídas ao longo de sua extensão. Entretanto, problemas de uniformidade de vazão ao longo dos tubos devidos à desuniformidade do tamanho dos poros e à redução da permeabilidade com o tempo de uso, podem torná-los inadequados para irrigação (Teeluck \& Sutton, 1998).

Apesar de todo o apelo ecológico apresentado por produtos oriundos de processos industriais que envolvem o reaproveitamento de resíduos, é imprescindível que estes sejam rigorosamente avaliados seguindo as recomendações técnicas vigentes a fim de que seja validada sua adequação para uso em sistemas de irrigação.

Os principais indicadores de desempenho de um emissor ou tubo emissor para microirrigação são coeficientes de variação de fabricação, susceptibilidade a obstrução, estabilidade temporal da relação vazão-pressão e sensibilidade à variação de temperatura e pressão (Souza et al., 2005; Frizzone et al., 2012). A caracterização hidráulica é fator primordial haja vista que o dimensionamento de um projeto de irrigação só é possível caso sejam conhecidas as informações técnicas do emissor, principalmente a relação vazão-pressão tal como, também, os parâmetros que possibilitem o cálculo da perda de carga.

Para o caso dos tubos porosos não há metodologias consagradas para caracterizá-los hidraulicamente, sobremaneira no que se refere à determinação dos parâmetros para cálculo da perda de carga. Por se tratar de um tubo com distribuição de vazão em marcha, além do fator de atrito da equação universal de perda de carga tem-se também o fator de redução de vazão (fator de Christiansen). Neste sentido, desenvolveu-se este trabalho com o intuito de caracterizar os tubos emissores porosos quanto aos aspectos de perda de carga, vazão em função da pressão e variação de vazão.

\section{Material e Métodos}

Os ensaios foram efetuados no primeiro semestre de 2011 sob a altitude de $575 \mathrm{~m}$ e latitude e longitude é de $22^{\circ} 42^{\prime}$ e $47^{\circ}$ 38 , respectivamente.

Os ensaios para determinação da curva vazão-pressão e do coeficiente de variação de fabricação foram realizados empregando-se um conjunto motobomba com rotação controlada por um inversor de frequência.

As pressões necessárias à condução dos ensaios $(29,4 ; 39,2$; 49,$1 ; 58,9 ; 68,7 ; 78,5$ e $88,3 \mathrm{kPa}$ ) foram obtidas por meio de um inversor de frequência observando-se o valor de pressão apresentado no manômetro digital (incerteza de medição de $0,25 \%$ ) instalado no início das linhas as quais foram constituídas por oito amostras de tubos Osmo-Drain com diâmetro interno de $12,88 \mathrm{~mm}$.

A estrutura de ensaio utilizada permitia o acoplamento simultâneo de quatro linhas de tubos com $2,50 \mathrm{~m}$ de comprimento. Em cada uma dessas linhas foram instaladas duas calhas coletoras com comprimento de $1,00 \mathrm{~m}$ e espaçadas em $0,25 \mathrm{~m}$, sendo coletado o volume de água referente ao comprimento de $1,00 \mathrm{~m}$ de tubo (Figura 1); apesar disto, a bancada de ensaio era composta por um reservatório de água fechado bombeado em circuito fechado passando por um filtro de tela de 400 mesh, a fim de evitar problemas de obstrução dos poros do tubo.

A determinação da vazão em cada uma das amostras foi feita conforme a Eq. 1 em que o cálculo da massa específica da água, em função da temperatura, foi efetuado por meio da Eq. 2 sugerida por Kell (1975).

$$
\mathrm{q}=\frac{\mathrm{m}_{\text {total }}-\mathrm{m}_{\text {coletor }}}{\rho \mathrm{t}} 3,6 \times 10^{6}
$$

$$
\begin{aligned}
\rho & =\left(999,8676+17,801161 \mathrm{~T}-7,942501 \times 10^{-3} \mathrm{~T}^{2}-\right. \\
& -52,56328 \times 10^{-6} \mathrm{~T}^{3}+137,6891 \times 10^{-9} \mathrm{~T}^{4}- \\
& \left.-364,4647 \times 10^{-12} \mathrm{~T}^{5}\right) /\left(1+17,735441 \times 10^{-3} \mathrm{~T}\right)
\end{aligned}
$$

em que:

q - vazão por unidade de comprimento de tubo, $\mathrm{L} \mathrm{h}^{-1} \mathrm{~m}^{-1}$

$\mathrm{m}_{\text {total }}$ - massa do coletor + massa de água coletada, $\mathrm{kg}$

$\mathrm{m}_{\text {coletor }}$ - massa do coletor, $\mathrm{kg}$

$\rho \quad$ - massa específica da água para dada temperatura, $\mathrm{kg} \mathrm{m}^{-3}$

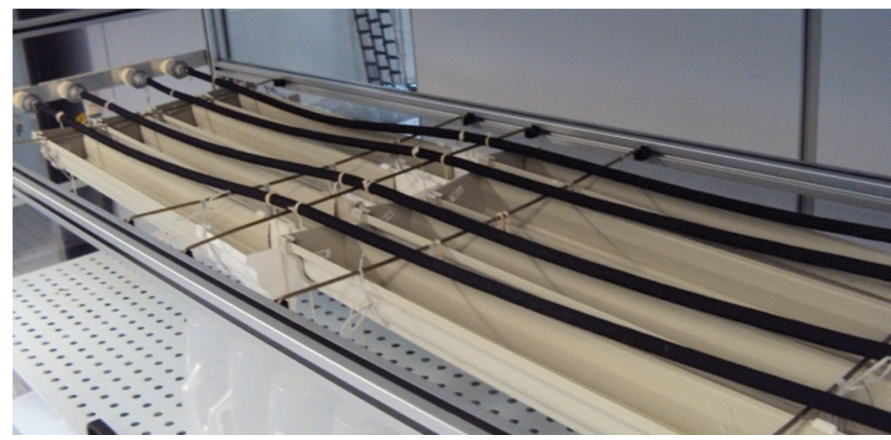

Figura 1. Estrutura utilizada para a realização do ensaio vazão-pressão dos tubos Osmo-Drain ${ }^{\oplus}$ 
t $\quad$ - tempo de ensaio, $s$

$\mathrm{T} \quad$ - temperatura, ${ }^{\circ} \mathrm{C}$

As variáveis temperatura, massa e tempo foram mensuradas por meio de termômetro $\left( \pm 1^{\circ} \mathrm{C}\right)$, balança digital $( \pm 0,1 \mathrm{~g})$ e cronômetro $( \pm 0,01 \mathrm{~s})$ respectivamente; a temperatura média da água durante os ensaios foi de $27,2{ }^{\circ} \mathrm{C}$.

$\mathrm{O}$ coeficiente de variação de fabricação (CVF) foi determinado com base nos dados coletados do ensaio vazão-pressão.

A viscosidade da água em função da temperatura foi calculada por meio da Eq. 3 resultante da aplicação do modelo sugerido por Likhachev (2003) aos dados empíricos apresentados por Azevedo Netto (2005).

$$
v=\frac{32,025666 \times 10^{-6}}{\rho} \mathrm{e}^{\frac{482,134866}{\mathrm{~T}+119,886026}}
$$

em que:

$v \quad$ - viscosidade cinemática, $\mathrm{m}^{2} \mathrm{~s}^{-1}$

Como o tubo poroso tem comportamento diferente dos emissores convencionais, realizou-se a modelagem da curva vazão-pressão considerando-se, para isto, um tubo hipotético, como o apresentado na Figura 2.

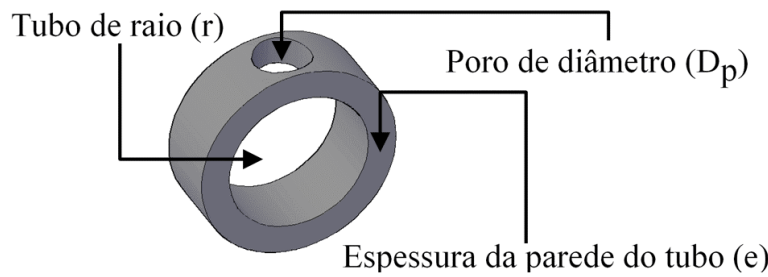

Figura 2. Desenho esquemático do tubo poroso ressaltando-se apenas um poro hipotético, em que Dp: diâmetro do poro; r: raio do tubo e espessura da parede do tubo

Com base de que o fator de atrito da equação de perda de carga universal pode ser representado por um modelo potencial (Eq. 4) (Yildirim, 2007; Rettore Neto et al., 2009) e que a pressão no interior do tubo é dissipada na forma de perda de carga, a vazão unitária do tubo poroso pode ser calculada por meio da Eq. 5.

$$
\begin{gathered}
f=a R^{b} \\
q=K_{p} \frac{5+b}{2+b}\left(\frac{P}{e}\right)^{\frac{1}{2+b}}
\end{gathered}
$$

em que:

f - fator de atrito

a, b - parâmetros de ajuste do modelo potencial

$\mathrm{R}$ - número de Reynolds, adimensional

$\mathrm{q}$ - vazão unitária, $\mathrm{m}^{3} \mathrm{~s}^{-1} \mathrm{~m}^{-1}$

$\mathrm{D}_{\mathrm{p}}$ - diâmetro do poro, $\mathrm{m}$

$\mathrm{P}^{\mathrm{p}}$ - pressão no interior do tubo, $\mathrm{Pa}$ e - espessura da parede, $\mathrm{m}$

K - constante

Como o tubo poroso pode sofrer alteração do seu diâmetro devido à pressão interna do tubo, uma vez que são tubos elásticos (Rettore Neto et al., 2013; 2014) pode-se inferir que o diâmetro dos poros também é passível de ser alterado (Eq. 6). Esta variação pode ser calculada por meio da equação de Hooke (Eq. 7) válida para o caso de um sólido solicitado por três tensões $\left(\sigma_{\mathrm{x}}, \sigma_{\mathrm{y}}, \sigma_{\mathrm{z}}\right)$ normais entre si (Nash, 1990).

$$
\begin{gathered}
\Delta \mathrm{D}_{\mathrm{p}}=\mathrm{k}_{1} \Delta \mathrm{C} \rightarrow \mathrm{D}_{\mathrm{p}}=\mathrm{D}_{\mathrm{p} 0}+\mathrm{k} \Delta \mathrm{C} \\
\varepsilon_{\mathrm{x}}=\frac{1}{\mathrm{E}}\left(\sigma_{\mathrm{x}}-\mathrm{v}\left(\sigma_{\mathrm{y}}+\sigma_{\mathrm{z}}\right)\right)
\end{gathered}
$$

em que:

$\Delta \mathrm{Dp}$ - variação do diâmetro dos poros, $\mathrm{m}$

$\mathrm{D}_{\mathrm{p} 0}$ - diâmetro do poro para pressão atmosférica, $\mathrm{m}$

$\Delta \mathrm{C}$ - variação do perímetro do cilindro, $\mathrm{m}$

C - perímetro do cilindro, $m$

$\mathrm{k}_{1} \quad$ - constante de proporcionalidade

$\varepsilon_{\mathrm{x}} \quad$ - deformação do sólido na direção $\mathrm{x}$, adimensional

$\sigma_{x}, \sigma_{y}, \sigma_{z}$ - tensões nas direções $x, y, z$, respectivamente, $\mathrm{Pa}$

$\mathrm{E}$ - módulo de elasticidade do material do qual o sólido é composto, $\mathrm{Pa}$

$v \quad$ - coeficiente de Poisson, adimensional

No caso do tubo e se tomando a direção x correspondente à direção circunferencial (c), tem-se: $\sigma_{x}=\sigma_{c}=\operatorname{Pr} / e, \sigma_{x}=\sigma_{c}=\operatorname{Pr} / e$; $\sigma_{\mathrm{y}}=\sigma_{1}=\operatorname{Pr} / 2 \mathrm{e} ; \sigma_{\mathrm{z}}=\sigma_{\mathrm{e}}=0$; já quando se considera a direção $\mathrm{x}$ correspondente à direção normal à parede do cilindro (e), temse: $\sigma_{\mathrm{x}}=\sigma_{\mathrm{e}}=0 ; \sigma_{\mathrm{y}}=\sigma_{\mathrm{c}}=\operatorname{Pr} / \mathrm{e} ; \mathrm{e}, \sigma_{\mathrm{z}}=\sigma_{\mathrm{l}}=\operatorname{Pr} / 2 \mathrm{e}$ (Norton, 2006); assim, o diâmetro dos poros e a espessura da parede do tubo, em função da pressão, são dados pelas Eqs. 8 e 9, respectivamente.

$$
\begin{gathered}
D_{p}=k_{2} P+k_{3} \\
e=k_{4}-k_{5} P
\end{gathered}
$$

em que:

$\mathrm{k}_{2}, \mathrm{k}_{3}, \mathrm{k}_{4}, \mathrm{k}_{5}$ - constantes

Aplicando as Eqs. 8 e 9 na Eq. 5, tem-se:

$$
\mathrm{q}=\left(\mathrm{k}_{2} \mathrm{P}+\mathrm{k}_{3}\right)^{\frac{5+\mathrm{b}}{2+\mathrm{b}}}\left(\frac{\mathrm{P}}{\mathrm{k}_{4}-\mathrm{k}_{5} \mathrm{P}}\right)^{\frac{1}{2+\mathrm{b}}}
$$

Seja $\mathrm{x}=(1 / 2+\mathrm{b}) \rightarrow(5+\mathrm{b} / 2+\mathrm{b})=3 \mathrm{x}+1$, tem-se a Eq. 11, que foi utilizada para representar a curva vazão-pressão do tubo poroso; os parâmetros da Eq. 11 foram ajustados utilizando-se o método dos mínimos quadrados.

$$
q=\left(k_{2} P+k_{3}\right)^{3 x+1}\left(\frac{P}{k_{4}-k_{5} P}\right)^{x}
$$


em que:

x - expoente de vazão

A determinação de perda de carga foi efetuada sob duas condições: com distribuição de vazão em marcha $\left(h f_{m}\right)$ e sem distribuição de vazão, ou seja, com tubo poroso impermeabilizado $\left(\mathrm{hf}_{\mathrm{s}}\right.$ ). Para ambas as condições foram utilizadas cinco amostras de tubo poroso com $18 \mathrm{~m}$ de comprimento efetuando-se a medição da perda de carga correspondente às velocidades aproximadas de entrada de 0,25 ; 0,$50 ; 0,75 ; 1,00 ; 1,50$ e $2,00 \mathrm{~m} \mathrm{~s}^{-1}$. Essas medidas foram feitas empregando-se um manômetro diferencial de mercúrio sendo a perda de carga calculada por meio das Eqs. 12 e 13.

A massa específica do mercúrio em função da temperatura, foi determinada conforme modelo sugerido por Holman \& Seldam (1994); a determinação da vazão foi efetuada por meio de um medidor eletromagnético com faixa de operação de 0 a $4 \mathrm{~m}^{3} \mathrm{~h}^{-1} \mathrm{e}$ incerteza de medição de $0,5 \%$, instalado a montante do tubo poroso.

$$
\begin{gathered}
\mathrm{hf}_{\mathrm{m} / \mathrm{s}}=\mathrm{h}\left(\frac{\rho_{\mathrm{hg}}-\rho}{1000}\right) \\
\rho_{\mathrm{hg}}=\frac{13595,08}{1+1,81886 \times 10^{-4} \mathrm{~T}}
\end{gathered}
$$

em que:

h - deflexão manométrica, $m$

$\rho_{\mathrm{hg}}$ - massa específica do mercúrio em função da temperatura, $\mathrm{kg} \mathrm{m}^{-3}$

Para determinação do fator de atrito efetuou-se a impermeabilização dos tubos com o uso de fita adesiva de silicone, aplicada sobre a superfície do tubo de modo a formar uma camada impermeável.

O cálculo do fator de atrito foi efetuado por meio do ajuste dos dados de perda de carga unitária em função da vazão conforme a Eq. 14; já os parâmetros da Eq. 4 (fator de atrito) foram calculados por meio das Eqs. 15 e 16.

$$
\begin{gathered}
J_{\mathrm{s}}=\mathrm{K}^{\prime} \mathrm{Q}^{\mathrm{m}} \\
\mathrm{a}=\left(\frac{\mathrm{v}}{4}\right)^{\mathrm{b}} \frac{\mathrm{K}^{\prime} \pi^{(2+\mathrm{b})} g \mathrm{gD}^{(5+\mathrm{b})}}{8} \\
\mathrm{~b}=\mathrm{m}-2
\end{gathered}
$$

em que:

$\mathrm{J}_{\mathrm{s}} \quad$ - perda de carga unitária, sob o regime permanente e uniforme, $\mathrm{m} \mathrm{m}^{-1}$

L - comprimento do tubo, $\mathrm{m}$

K' - constante

D - diâmetro do tubo, $\mathrm{m}$

Para caracterização do fator de redução de perda de carga (F) se efetuaram ensaios de perda de carga com o tubo poroso funcionando sem impermeabilização $\left(\mathrm{hf}_{\mathrm{s}}\right)$ ou seja, com distribuição de água sendo $\mathrm{F}$ calculado por meio da Eq. 17. Esses valores foram comparados com aqueles calculados por meio da equação de Christiansen (Eq. 18), considerando um número de saídas tendendo ao infinito, ou seja, o fator de perda de carga (F) tende a 1/(m+1) (Scaloppi \& Allen, 1993; Valiantzas, 2002).

$$
\begin{gathered}
\mathrm{F}=\frac{\mathrm{hf}_{\mathrm{m}}}{\mathrm{hf}_{\mathrm{s}}} \\
\mathrm{F}=\frac{1}{\mathrm{~m}+1}+\frac{1}{2 \mathrm{~N}}+\frac{\sqrt{\mathrm{m}-1}}{6 \mathrm{~N}^{2}}
\end{gathered}
$$

em que:

m - expoente da equação que caracteriza a perda de carga no conduto

$\mathrm{N}$ - número de emissores

Entretanto, como a vazão ao longo de uma tubulação com distribuição em marcha pode ser representada pela Eq. 19 (Yildirim \& Ağiralioğlu, 2004) isto é, a vazão unitária não é contante ao longo da tubulação, o F pode ser calculado por meio da Eq. 20.

$$
\begin{gathered}
\mathrm{Q}(1)=\mathrm{Q}\left(1-\frac{1}{\mathrm{~L}}\right)^{\beta} \\
\mathrm{F}=\frac{1}{\beta \mathrm{m}+1}
\end{gathered}
$$

em que:

1 - distância entre a entrada do tubo e um ponto qualquer do tubo, $\mathrm{m}$

$\mathrm{Q}(\mathrm{l})$ - vazão em um ponto qualquer do tubo, $\mathrm{m}^{3} \mathrm{~s}^{-1}$

$\mathrm{Q}$ - vazão na entrada, $\mathrm{m}^{3} \mathrm{~s}^{-1}$

L - comprimento total do tubo, $\mathrm{m}$

$\beta$ - constante.

\section{Resultados e Discussão}

$\mathrm{Na}$ Tabela 1 estão apresentados os resultados dos ensaios para determinação da curva vazão-pressão e coeficiente de variação de fabricação do tubo avaliado.

Segundo a ABNT (2006) o coeficiente de variação de fabricação de um emissor ou tubo emissor, seja ele regulado ou não, não deve ser superior a $7 \%$. Apesar desta norma não apresentar requisitos referentes a tubos porosos, pode-se observar que este atenderia aos critérios estabelecidos para os emissores convencionais em todas as pressões avaliadas; portanto com base no CVF, conclui-se que o material avaliado seria apto para uso em sistemas de irrigação apresentando qualidade de fabricação superior à do material descrito por Teeluck \& Sutton (1998).

Na Figura 3 está apresentado o ajuste da relação vazãopressão (Eq. 11) tendo este apresentado coeficiente de 
Tabela 1. Resultado da vazão unitária média (q) e coeficiente de variação de fabricação (CVF) para oito amostras de tubos Osmo-Drain ${ }^{\circledR}$ sob diferentes pressões avaliadas

\begin{tabular}{|c|c|c|c|c|c|c|c|}
\hline \multirow{3}{*}{ Amostra } & \multicolumn{7}{|c|}{ Pressão (kPa) } \\
\hline & 29,4 & 39,2 & 49,1 & 58,9 & 68,7 & 78,5 & 88,3 \\
\hline & \multicolumn{7}{|c|}{ Vazão $\left(L h^{-1} m^{-1}\right)$} \\
\hline 1 & 22,525 & 28,283 & 35,934 & 47,110 & 61,281 & 77,788 & 95,774 \\
\hline 2 & 20,924 & 25,317 & 32,157 & 41,800 & 54,357 & 69,212 & 83,290 \\
\hline 3 & 19,561 & 24,578 & 31,369 & 41,668 & 54,898 & 68,765 & 84,831 \\
\hline 4 & 19,750 & 25,154 & 31,792 & 42,039 & 54,987 & 70,290 & 87,245 \\
\hline 5 & 20,100 & 25,958 & 33,055 & 43,831 & 57,434 & 73,193 & 89,299 \\
\hline 6 & 19,406 & 24,416 & 30,987 & 40,866 & 53,533 & 67,403 & 82,970 \\
\hline 7 & 20,809 & 26,608 & 34,085 & 45,632 & 59,385 & 75,656 & 91,897 \\
\hline 8 & 20,308 & 25,427 & 32,390 & 42,884 & 55,858 & 70,473 & 85,451 \\
\hline$q\left(L^{-1} m^{-1}\right)$ & 20,423 & 25,718 & 32,721 & 43,229 & 56,467 & 71,597 & 87,594 \\
\hline CVF (\%) & 4,954 & 4,871 & 4,958 & 5,007 & 4,767 & 5,053 & 5,116 \\
\hline
\end{tabular}

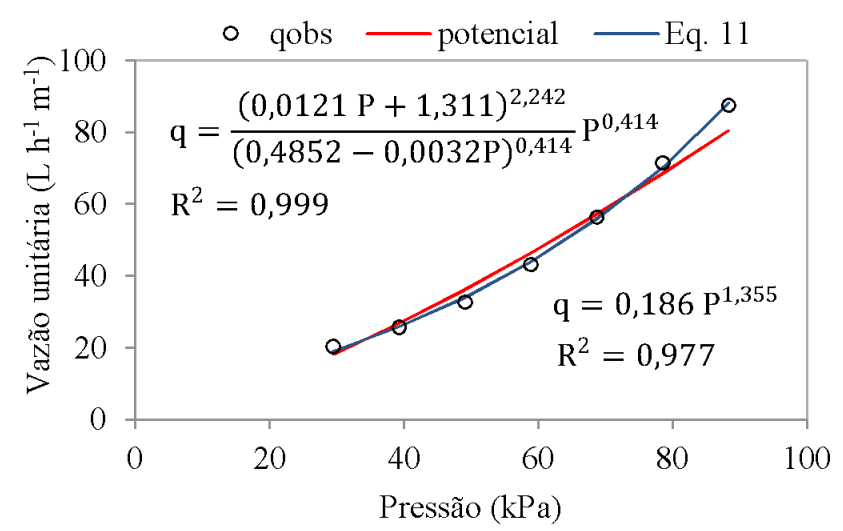

Figura 3. Relação entre a vazão unitária e a pressão interna do tubo Osmo-drain ${ }^{\odot}$

determinação de 0,99 . O expoente de vazão foi de 0,41 indicando que o regime de escoamento foi turbulento (Souza et al., 2005).

Para o caso dos emissores convencionais tem-se que a relação vazão pressão é dada por $\mathrm{q}=\mathrm{kP}$ (Souza et al., 2005; Yildirim, 2007; Almeida et al., 2009; Frizzone et al., 2012). Porém, para o caso em questão e devido à variação do diâmetro e do percurso dos poros mediante a variação de pressão interna do tubo, a relação vazão-pressão é alterada de modo que o modelo potencial $\left(\mathrm{q}=0,186 \mathrm{P}^{1,355}\right)$ apresentou ajuste com coeficiente de determinação inferior àquele obtido pelo modelo proposto $(\mathrm{q}$ $\left.=\left\{\left[(0,0121 \mathrm{P}+1,311)^{2,242}\right] /(0,4852-0,0032 \mathrm{P})^{0,414}\right\} \mathrm{P}^{0,414}\right)$, como pode ser visualizado na Figura 3.

$\mathrm{Na}$ Figura 4A estão apresentados os resultados de perda de carga unitária para o tubo sem distribuição em marcha (Js). Pode-se observar que o modelo potencial se ajustou aos dados, tendo um coeficiente de determinação de 0,99 .

Aplicando os parâmetros $\mathrm{K}=1,739019$ e $\mathrm{m}=2,240231$ da equação de perda de carga unitária apresentada na Figura 4A nas Eqs. 14 e 15, sendo o valor de $\mathrm{K}$ transformado para o caso em que a unidade vazão $\mathrm{em} \mathrm{m}^{3} \mathrm{~s}^{-1} \mathrm{e}$ considerando a temperatura média dos ensaios $\left(27,2^{\circ} \mathrm{C}\right)$ tem-se a Eq. 21 .

$$
\mathrm{f}=0,00798 \mathrm{R}^{0,240231}
$$

Como foram avaliadas cinco amostras que apresentaram variações construtivas entre elas, conforme demonstrado na Tabela 1, esperava-se que o coeficiente de atrito (f) apresentasse dispersão entre as amostras avaliadas (Figura 4C); no entanto e se considerando a média entre as mostras para o mesmo número de Reynolds, obteve-se elevada concordância entre os dados observados e os calculados por meio da Eq. 21.

Uma questão a ser analisada com maior critério é que o fator de atrito aumenta juntamente com o número de Reynolds; contrariamente, sabe-se que pelas leis da resistência no escoamento turbulento considerando a rugosidade relativa constante, o fator de atrito diminui na medida em que o número de Reynolds aumenta. Isto indica que, neste caso, o tubo analisado deve ter sofrido alterações em seu diâmetro devido à pressão interna do tubo tendo em vista que se trata de um tubo elástico (Rettore Neto et al., 2013; 2014).

Como os ensaios de perda de carga foram feitos com pressão de entrada constante, então a pressão ao longo do tubo para as vazões menores é maior uma vez que a perda de carga é menor; neste caso, o diâmetro médio do tubo também seria maior e, consequentemente, a rugosidade relativa diminui. Desta forma, pode-se inferir que a rugosidade relativa aumentou proporcionalmente com o número de Reynolds. Isto pode ser observado por meio da Figura 4D onde estão apresentados os valores calculados de rugosidade relativa. Esses cálculos foram feitos por meio da equação de Swamme, que é válida para todos os regimes de escoamento (Swamme, 1993).

O fator de redução de perda de carga (F) foi de 0,247 (coeficiente angular da reta de regressão apresentada na Figura 4B); aplicando a equação de Christiansen (Eq. 18) para um número de emissores tendendo ao infinito, tem-se que $\mathrm{F}=0,309$, que é aproximadamente $25 \%$ maior que o valor determinado. Isto se deve ao fato de que o fator de redução proposto por Christiansen parte do pressuposto de que a vazão distribuída ao longo da tubulação é constante, ou seja, a vazão de escoamento ao longo da tubulação tem uma relação linear com a extensão do tubo (Scaloppi \& Allen, 1993; Valiantzas, 2002).

Quando se considera a variação da vazão, o modelo potencial, ao longo da tubulação, o fator F calculado por meio da Eq. 20, torna-se equivalente ao $\mathrm{F}$ determinado empiricamente para $\beta=1,3615$, ou seja, a vazão ao longo do tubo pode ser expressa conforme a Eq. 22. 
A.

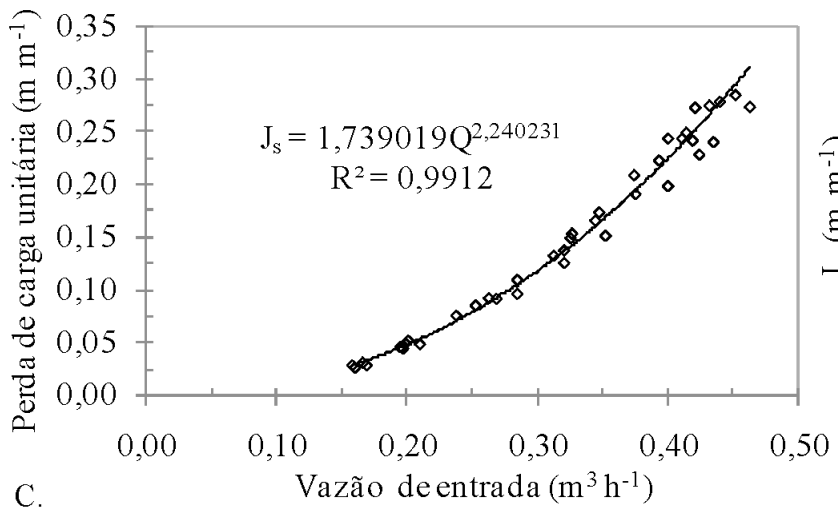

C.

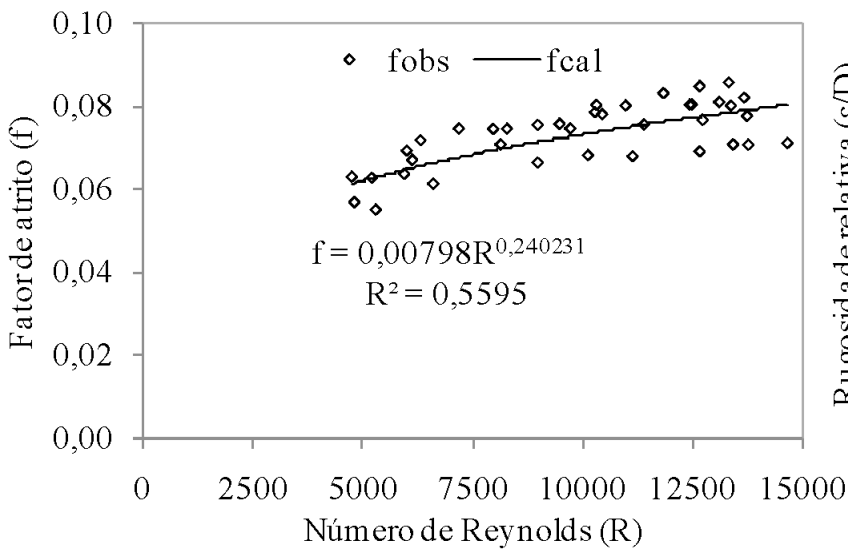

B
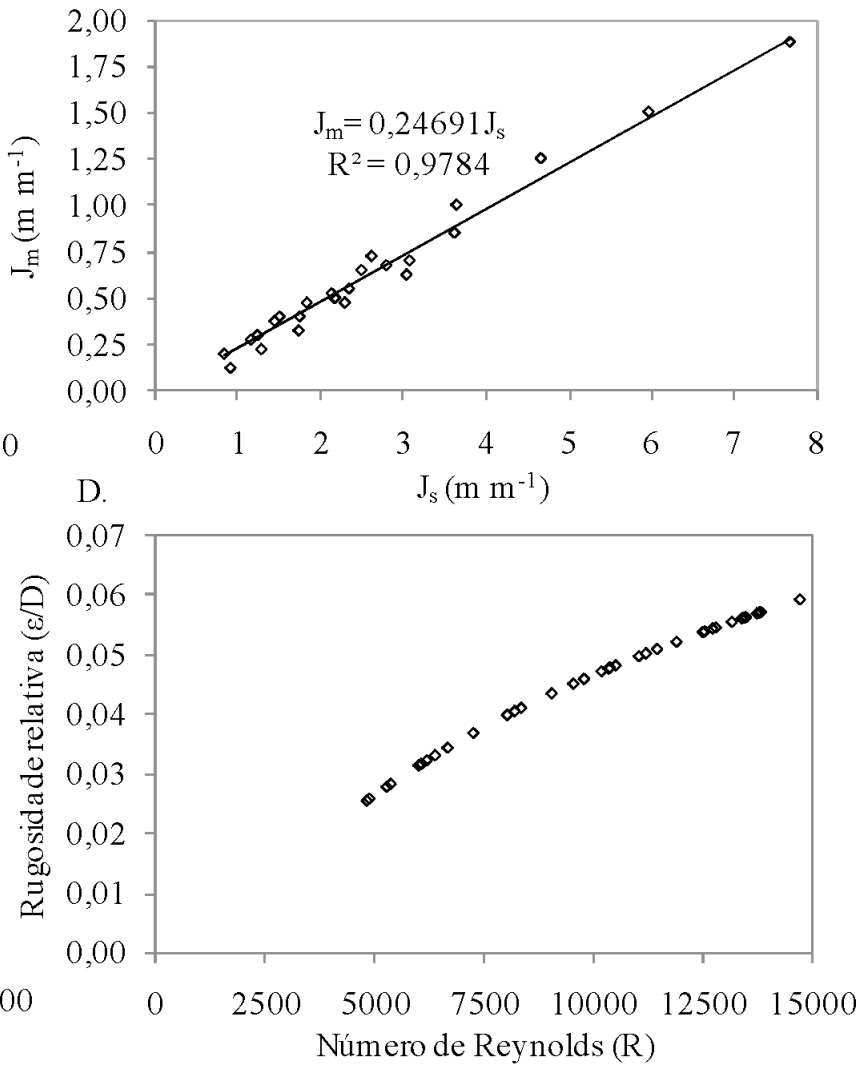

Figura 4. (A) Perda de carga observada com o tubo impermeabilizado; (B) Relação entre a perda de carga unitária para o tubo com distribuição de vazão e o tubo impermeabilizado; (C) Fator de atrito observado (fobs) e calculado (fcal); (D) rugosidade relativa em função do número de Reynolds

$$
Q(1)=Q\left(1-\frac{1}{L}\right)^{1,3615}
$$

\section{Conclusões}

1. O tubo poroso avaliado apresentou bom desempenho, no que se refere ao coeficiente de variação de vazão para todas as pressões analisadas.

2. O fator de atrito apresentou comportamento divergente da literatura de modo que outras fontes de variação podem ter interferido nos resultados, sendo essas fontes de variação atribuídas à variação do diâmetro em consequência da pressão interna no tubo.

3. O fator de redução de perda de carga difere dos valores recomendados para linhas laterais convencionais tendo em vista que a distribuição de vazão é contínua.

4. O modelo desenvolvido para representação da curva vazão-pressão representou adequadamente os dados empíricos sendo que o modelo potencial não se ajustou adequadamente.

\section{Agradecimentos}

Os autores agradecem ao Ministério da Ciência e Tecnologia (MCT), à Coordenação de Aperfeiçoamento de Pessoal de Nível Superior (CAPES), ao Conselho Nacional de Desenvolvimento
Científico e Tecnológico (CNPq) e à Fundação de Amparo à Pesquisa do Estado de São Paulo (FAPESP) pelo apoio financeiro a esta pesquisa através do Instituto Nacional de Ciência e Tecnologia em Engenharia da Irrigação (INCTEI). À empresa Samaritá Indústria e Comércio Ltda. por ter fornecido o material necessário à condução desta pesquisa.

\section{Literatura Citada}

ABNT - Associação Brasileira de Normas Técnicas. Equipamentos de irrigação agrícola - Emissores e tubos emissores - Especificações e métodos de ensaio. ABNT NBR ISO 9261. São Paulo: ABNT, 2006. 17p.

Almeida, C. D. G. C.; Botrel, T. A.; Smith, R. J. Characterization of the microtube emitters used in a novel micro-sprinkler. Irrigation Science, v.27, p.209-214, 2009.

Azevedo Netto, J. M.; Fernandez, M. F.; Araújo, R.; Ito, A. E. Manual de hidráulica. São Paulo: Edgard Blücher Ltda, 2005. 669p.

Bertollo, S. A. M.; Fernandes Júnior, J.; Villaverde, R. B.; Migotto Filho, D. Pavimentação asfáltica: Uma alternativa para a reutilização de pneus usados. Revista de Limpeza Pública, v.54, p.23-30, 2000.

Frizzone, J. A.; Freitas, P. S. L.; Rezende, R.; Faria, M. A. Microirrigação: Gotejamentoemicroaspersão. Maringá: Eduem, 2012. 356p. 
Holman, G. J. F.; Seldam, C. A. ten. A critical evaluationof the thermophysical properties of mercury. Journal of Physical and Chemical Reference Data, v.23, p.807-827, 1994.

Kell, G. S. Density, thermal expansivity, and compressibility of liquid water from $0^{\circ}$ to $150^{\circ} \mathrm{C}$. Journal of Chemical Engineering Data, v.20, p.97-105, 1975.

Lagarinhos, C. A. F.; Tenório, J. A. S. Tecnologias utilizadas para a reutilização, reciclagem e valorização energética de pneus no Brasil. Polímeros: Ciência e Tecnologia, v.18, p.106-118, 2008.

Likhachev, E. R. Dependence of water viscosity on temperature and pressure. Technical Physics, v.48, p.514-515, 2003.

Motta, F. G. A cadeia de destinação dos pneus inservíveis - O papel da regulação e do desenvolvimento tecnológico. Ambiente e Sociedade, v.11, p.167-184, 2008.

Nash, W.A. Resistência dos materiais. São Paulo: Makron,1990. $521 \mathrm{p}$.

Norton, R. L. Projetode máquinas: uma abordagem integrada. Porto Alegre: Bookman, 2006. 931p.

Osmo-Drain. http://osmo-drain.de/en/index.php. 1Dez. 2012.

Qiaosheng, S.; Zuoxin, L.; Zhenying, W.; Haijun, L. Simulation of the soil wetting shape under porous pipesub-irrigation using dimensional analysis. Irrigation and Drainage, v.56, p.389-398, 2007.

Rettore Neto, O.; Botrel, T. A. ; Frizzone, J. A. ; Camargo, A. P. Method for determining friction head loss along elastic pipes. Irrigation Science, v.32, p.329-339, 2014.
Rettore Neto, O.; Botrel, T. A.; Frizzone, J. A.; Pinto, M. F.; Camargo, A. P. Quantificação do erro na determinação da perda contínua de carga em tubos elásticos. Engenharia Agrícola, v.33, p.1312-1321, 2013.

Rettore Neto, O; Frizzone, J.A.; Miranda, J.H.; Botrel,T. A. Perda de carga localizada em emissores não coaxiais integrados a tubos de polietileno. Engenharia Agrícola, v.29,p.28-39, 2009.

Scaloppi, E. J.; Allen, R. G. Hydraulics of irrigation laterals: comparative analysis. Journal of Irrigation and Drainage Engineering, v.119, p.91-115, 1993.

Souza, J. A. R.; Denículi, W. Batista, R. O.; Val, J. C. C.; Matos, A. T. Caracterização hidráulica de microaspersor aplicando água limpa, água residuária de avicultura e de bovinocultura. Engenharia na Agricultura, v.13, p.161-172, 2005.

Swamme, K. P. Design of a submarine oil pipeline. Journal of transportation Engineering, v.119, p.159-170, 1993.

Teeluck, M.; Sutton, B.G. Discharge characteristics of a porous pipe microirrigation lateral. Agricultural Water Management, v.38, p.123-134, 1998.

Valiantzas, J. D. Continuous outflow variation along irrigation laterals: Effectof the number of outlets. Journal of Irrigation and Drainage Engineering, v.128, p.34-42, 2002.

Yildirim, G. An assessment of hydraulic design of trickle laterals considering effect of minor losses. Irrigation and Drainage, v.56, p.399-421, 2007.

Yildirim, G. M. A.; Ağiralioğlu, N. Comparative analysis of hydraulic calculation methods in design of microirrigation laterals. Journal of Irrigation and Drainage Engineering, v.130, p.201-217, 2004. 